\title{
The left angular gyrus is causally involved in information buffering and context formation: evidence from a narrative reading task
}

Running title: A causal role for left Ag in context formation

\section{Francesca M. BRANZI ${ }^{1}$, Gorana POBRIC ${ }^{2}$, JeYoung JUNG ${ }^{3}$, and Matthew A. LAMBON RALPH $^{1}$}

\author{
${ }^{1}$ MRC Cognition \& Brain Sciences Unit, University of Cambridge, Cambridge, UK, CB2 7EF \\ ${ }^{2}$ School of Biological Sciences, University of Manchester, Manchester, UK, M13 9PL \\ ${ }^{3}$ School of Psychology, University of Nottingham, Nottingham, UK, NG7 2RD
}

\section{Address for correspondence:}

Dr. Francesca M. Branzi, E: Francesca.Branzi@mrc-cbu.cam.ac.uk. or

Prof. Matthew A. Lambon Ralph, E: Matt.Lambon-Ralph@mrc-cbu.cam.ac.uk.

Number of pages: 28

Number of figures: 4

Number of tables: 2

Number of words for Abstract: 174

Number of words for Introduction: 650

Number of words for Discussion: 1095

Conflict of Interest: The authors declare no competing financial interests.

Acknowledgments: this research was supported by a Postdoctoral Fellowship from the European Union's Horizon 2020 research and innovation programme, under the Marie Sklodowska-Curie grant agreement No 658341 to FMB; an ERC Advanced Grant (GAP: 670428 - BRAIN2MIND_NEUROCOMP) and MRC Programme Grant (MR/R023883/1) to MALR and a Beacon Anne McLaren Research Fellowship (University of Nottingham) to JJ. 


\begin{abstract}
The role of the left angular gyrus (Ag) in semantic processing remains unclear. In this study, we used transcranial magnetic stimulation (TMS) to test the hypothesis that the left Ag supports the information buffering processes necessary for context-dependent semantic integration (narrative reading task). We applied online TMS to the left Ag to disrupt the buffering processes while female and male human participants integrated information between two paragraphs of text presented sequentially (i.e., context and target conditions). We assessed the effect of TMS on the left Ag by recording reading times for target conditions during the reading task and by asking participants to retrieve contextual information given the target condition as cue in a successive memory task. TMS applied over the left Ag during the reading task impaired the retrieval of contextual information in the memory task without affecting reading times. These results suggest that the Ag supports information buffering and context formation. This study provides the first evidence of a causal role of the left Ag in language tasks that require context-dependent semantic integration.
\end{abstract}

Significance statement: The present study offers novel insights into the processes that are associated with the left angular gyrus (Ag) activation during context-dependent naturalistic language processing. We used TMS while human participants read written narratives to interfere with online language integration. We measured how TMS over the left Ag was affecting reading times and retrieval of integrated narrative representations. We were able to show that the left $\mathrm{Ag}$ is causally involved in information buffering and context formation. 


\section{Introduction}

The left angular gyrus (Ag) is implicated in a range of cognitive activities (Seghier, 2013; Humphreys and Lambon Ralph, 2015). Amongst these, its specific role during semantic tasks is still under debate. Some have proposed that the left Ag might play a key role in the integration of semantic information (Humphries et al., 2007; Binder et al., 2009), while others have argued that left Ag supports buffering processes that operate on content that is integrated elsewhere (Vilberg and Rugg, 2012; Humphreys and Lambon Ralph, 2015, 2017). The latter hypothesis is in accord with evidence showing that left $\mathrm{Ag}$ is engaged when semantic tasks require context-dependent integration (Simony et al., 2016; Baldassano et al., 2017; van der Linden et al., 2017; Branzi et al., 2019), but deactivated when tasks do not require such process (Humphreys et al., 2015; Humphreys and Lambon Ralph, 2015). This transcranial magnetic stimulation (TMS) study tested the hypothesis that, during semantic tasks, the primary functional role of the left Ag reflects information buffering and context formation.

TMS was applied over the left Ag while human participants read narratives composed of two consecutive passages (context and target conditions). We hypothesised that if the left Ag is necessary for integrating context and target information, Ag TMS applied between context and target presentation should affect the encoding of a context-target integrated representation. To test this, after the reading task, participants performed a memory task. Importantly, Ag activity is positively engaged during encoding and predictive of better performance at memory retention only when the buffered information fits with a knowledge-based schema (Humphreys and Lambon Ralph, 2015; van der Linden et al., 2017). Hence, an Ag TMS effect should be observed only when context and target passages are semantically coherent (i.e., same schema), but not when their integration requires context updating. To test this, Ag TMS was applied during two different types of narrative conditions in which the same target paragraph was preceded by different types of context: (i) a highly congruent context (HC) which maximised the information contained in a coherent semantic gestalt; (ii) a low-congruent (LC) paragraph with a divergent meaning, thus requiring updating the semantic context. In the memory 
task, participants were presented with the target condition (both $\mathrm{HC}$ and LC) as a cue and required to retrieve context-related information ("Cue Target” trials). We expected Ag TMS to impair behavioural performance in HC conditions, but to have no effect on the retrieval of LC conditions.

Furthermore, memory representations that include elements following and preceding the update of information, are harder to retrieve than representations that require retrieval of associations within the same event (Speer and Zacks, 2005; Swallow et al., 2009). Accordingly, we expected to find increased response times (RTs) and error rates for low over high context-to-target congruency under standard conditions (i.e., no Ag TMS) and that Ag TMS would diminish this effect given the Ag's role in information buffering and context formation. To rule out the possibility that TMS was affecting encoding in general, rather than the encoding of context-target integrated representations specifically, we employed a control condition in the memory task. In "Cue Context trials", participants were required to retrieve the same context-related information as in the Cue Target conditions. However, they were provided with some contextual information as cue.

Finally, if the left $\mathrm{Ag}$ is necessary for integrating information between context and target, $\mathrm{Ag}$ TMS between context and target presentation during reading should affect also reading times locked to the target presentation. Similarly as in the memory task, we expected this effect for HC conditions only (van der Linden et al., 2017). Besides HC and LC conditions, in the reading task we also included (iii) a no context control condition (NC) in which the same target was preceded by a number reading task. This control condition allowed verification that TMS effects measured during the reading task were attributable to the buffering of contextual information only. 


\section{Materials and Methods}

\subsection{Participants}

Eighteen volunteers took part in the study (average age $=22$, standard deviation $(\mathrm{SD})=3 ; \mathrm{N}$ female $=12$ ). All participants were right-handed (Oldfield, 1971), native English speakers with no history of neurological or psychiatric disorders and normal or corrected-to-normal vision. Written informed consent was obtained from all participants. The experiment was approved by the local ethics committee.

\subsection{Stimuli}

Reading task. The experimental stimuli used in the reading task were the same as in Branzi et al. (2019). Thus, a total of 40 narrative pairs, each one composed by two paragraphs, were employed in the reading task. For each narrative pair, the same second paragraph (target) was preceded by different first paragraphs (contexts) that could be either high-congruent (i.e., HC) or low-congruent (i.e., LC) with the target in terms of meaning. Both HC and LC context paragraphs could be integrated with the targets paragraphs, though a reworking of the evolving semantic context was required after LC contexts, because of a shift in the semantic context (see $\underline{\text { Table } 1}$ for an example of the stimuli; for the full list of the stimuli used in the reading task see Branzi et al., 2019). Homonym words (e.g., bank), presented at the beginning of the target paragraph, were employed to determine the exact point in the paragraph in which the shift in the semantic context should have been experienced. Finally, the $\mathrm{NC}$ condition, where the target (the same as in HC and LC conditions) was preceded by a string of numbers, was employed as a control condition. In fact, the NC condition allowed to verify that any effect of TMS measured for HC and LC conditions was due to buffering processes, and not to bottomup attention triggered by the presentation of the target paragraph. 
Please, insert here Table 1

Memory task. The experimental stimuli employed in the memory task were 75 items, consisting in (1) the presentation of a "cue" displayed on the screen followed by (2) the presentation of a question relative to the context part of the narrative along with three possible answers (Q\&As, see Table 2 for some examples). Importantly, in the memory task there were different types of conditions. These conditions did not differ in respect to the type of information that participants had to retrieve (the same contextual information), rather they differed in the type of cue. In one condition the cues only corresponded to the information in the target paragraph [i.e., HC Cue Target conditions $(\mathrm{n}=25)$ and LC Cue Target conditions $(n=25)]$, and hence tested participants' ability to retrieve target-context integrated representations. By contrast in the other condition, the cues contained contextual information [i.e., Cue Context conditions $(n=25)]$ and thus did not require participants to retrieve the integrated meaning of the two sequential paragraphs. The type of contextual information that participants were required to retrieve during the memory task corresponded to a variety of episodic details (e.g., who, where, when) (see Table 2).

Please, insert here Table 2 


\subsection{Task procedures}

Before starting the experimental study, all participants signed an informed consent and were given written and oral instructions. A PC running ePrime (Psychology Software Tools, Inc., Pittsburgh, PA) was used to present the items and record participants' behavioural responses. The participants completed three sessions on different days. During two out of three sessions, participants received TMS (TMS over the left Ag and TMS over the Vertex). In one session, TMS was not used. The sessions were at least 1 week apart (average days between sessions $=12, \mathrm{SD}=3$ ) and the order of sessions was counterbalanced across participants.

In each session, each participant performed two different tasks: a reading task and a memory task, both divided in two parts. Specifically, each session started with a reading task, in which participants had to read half of the narratives. The reading task was immediately followed by a memory task, comprised of questions about the narratives presented in the reading task. After a short break (10 minutes), the remaining narratives were presented in a second reading task, which was followed by another memory task (including questions relating to the narratives presented in the second reading task).

During the TMS sessions, online TMS was delivered during each trial in the reading tasks (for detail see Figure 1 and Stimulation Parameters and Stimulation Sites). TMS was not applied during the memory tasks. We did not apply TMS during the memory task since the goal of the present study was to measure the contribution of the left Ag for semantic integration during comprehension. Since the left Ag is implicated not only in language processing but also in memory retrieval (Rugg and Vilberg, 2013; Humphreys and Lambon Ralph, 2015), not applying TMS during the memory task ensured that any observed TMS effect reflected the contribution of Ag for integrating semantic information during the reading task. 


\section{Please, insert here Figure 1}

At the end of each TMS session, participants completed a questionnaire in which they reported the extent to which TMS was perceived as uncomfortable and distracting (scales from $1=$ not very, to 7 = very). The scores obtained were overall low (Ag distracting: average $=3.8, \mathrm{SD}=1.3 ; \mathrm{Ag}$ uncomfortable: average $=3.9, \mathrm{SD}=1.3 ;$ Vertex distracting: average $=3.3, \mathrm{SD}=1.2 ;$ Vertex uncomfortable: average $=3, \mathrm{SD}=1.4$ ), suggesting that the TMS protocol induced only minimum discomfort and distractibility during the task. A trend toward significance indicated that overall TMS over the Ag obtained slightly higher scores on both scales [main effect of Site: $F(1,17)=4.185, p=$ 0.057, $\left.\eta \mathrm{p}^{2}=0.198\right]$. Finally, lack of significant Site $\times$ Type of Scale interaction $[\mathrm{F}(1,17)=1.034, \mathrm{p}$ $\left.=0.323, \eta \mathrm{p}^{2}=0.057\right]$ suggested that TMS on both sites was inducing similar ratings for discomfort and distractibility.

\section{Memory task.}

There were 25 items per condition (Cue Context, LC Cue Target and HC Cue Target). The LC Cue Target trials (25) were presented with half of the Cue Context trials (12 or 13), always after reading the LC narratives. The HC Cue Target trials (25) were presented with the remaining Cue Context trials (13 or 12), always after the HC narratives. The memory task was a 3 alternative-forced-choice task. Each trial started with the presentation of a "cue" displayed on the screen until participants made a button response, which was followed by the presentation of a question and 3 possible responses (Q\&Rs). The Q\&Rs were displayed until the participants made their selection by button response, up to a time limit of 8.5 seconds (s). Rest time (black fixation cross) was presented between cue and Q\&Rs and between trials (fixed time intervals: 250ms and 2000ms, respectively). Finally, whilst the 
stimuli presented in the reading task were the very same across all the three sessions, those presented during the memory task were always different across the three sessions.

Reading task.

In the reading task, there were 40 items per condition (HC, LC and NC). As in our previous study (Branzi et al., 2019), each trial consisted in the presentation of two paragraphs of text (context and target) that participants had to read silently (verbal material and numbers). Contexts and targets were displayed on the screen until participants pressed a button to indicate that they had finished reading the paragraph (for both contexts and targets). The instruction emphasized speed, given the limited amount of time for reading [max. duration for context was $12 \mathrm{~s}$ and for targets was $7.5 \mathrm{~s}$ ], but also the need to understand and encode the information presented in the narratives. We informed participants that at the end of each reading task they would perform a memory task, requiring them to answer questions on the content of the narratives. We also specified that in order to perform the task it would be necessary to integrate the information presented in contexts and targets. Rest time was varied between context and targets [range between 500 milliseconds (ms) and 1200ms, average time $=820 \mathrm{~ms}$ ] and between trials (range between $3000 \mathrm{~ms}$ and $5460 \mathrm{~ms}$, average time $=4230 \mathrm{~ms}$ ) during which a black fixation cross was presented.

HC and LC trials were presented separately. Given that HC and LC conditions were terminating with the same target paragraph, mixing them during the reading task would have created confusion during the memory task. Thus, the first reading task in each session included either HC trials (40) or LC trials (40), and half of the NC trials (20). The second reading task included the remaining narratives (either LC trials or HC trials, and the remaining NC trials). The order of presentation of LC and HC conditions was counterbalanced across participants and sessions. 


\section{Stimulation Parameters and Stimulation Sites.}

TMS was delivered using a Magstim Rapid2 stimulator (Magstim Co., Whitland, UK) and a figure-of-eight coil with a diameter of $70 \mathrm{~mm}$. Stimulation was performed at $120 \%$ of the individual's motor threshold, measured before the start of the first session (mean stimulation intensity $=71, \mathrm{SD}=$ 8 , range $=54-84)$. The resting motor threshold of the relaxed contralateral abductor pollicis brevis muscle was measured as the lowest stimulation intensity able to cause a visible twitch in the muscle 5 out of 10 times (Sandrini et al., 2011).

For each trial, one train of five pulses $(10 \mathrm{~Hz}$ for $500 \mathrm{~ms})$ was delivered. This stimulation protocol has already been used over the parietal cortex to induce inhibitory effects during language processing (Sliwinska et al., 2015). Unlike previous studies, the first pulse was administered before the presentation of the target (see Figure 1), to avoid disrupting reading (TMS to the Ag can induce eye twitches). A pilot study confirmed that this procedure was successful for obtaining the expected TMS effects. The rest time between the context and target phases was variable and randomized (see Figure 1). Thus, participants were not able to anticipate when they would receive TMS. Finally, the TMS frequency, intensity and duration were well within established international safety limits (Rossini et al., 2015).

The stimulation site for the left Ag region corresponded to the MNI coordinates $(x=-48, y=$ $-63, \mathrm{z}=36$ ) derived from our previous fMRI study, in which the same experimental material was used and where Ag activity was modulated by context integration (Branzi et al., 2019) (see Figure 2). During the Ag TMS testing session, a Polaris Vicra infrared camera (Northern Digital, Waterloo, ON, Canada) was used in conjunction with the Brainsight frameless stereotaxy system (Rogue Research, Montreal, QC, Canada) to register the participant's head to their own MRI scan to accurately target stimulation throughout the experiment. The location of the control site, i.e., Vertex, was established for each participant by using the international 10-20 system (Steinmetz et al., 1989). The halfway 
intersection of the two lines was marked using a skin marker and corresponded to individual Vertex control site.

\section{Please, insert here Figure 2}

\subsection{Data analysis}

\section{Behavioural data analyses}

Memory Task.

Behavioural analyses were performed on RTs and accuracy measures. RT analysis was conducted only for correct trials. Moreover, after having eliminated error trials, also trials exceeding 3 SDs above or below a given participant's mean were excluded from the RT analyses, causing an overall loss of $2 \%$ of data, across all task conditions and type of sessions. Then, we conducted two separate $3 \times 3$ within-subject ANOVAs (one for RTs and one for accuracy measures) with the repeatedmeasures factors condition (NC, LC and HC) and session-type (no TMS, TMS to left Ag, and TMS to vertex). Bonferroni correction for multiple comparisons was applied on post hoc pairwise contrasts.

Reading Task.

Behavioural analyses were performed on reading times only. In fact, since participants were reading silently, in this task it was not possible to measure error rates. First, trials exceeding 3 SDs above or below a given participant's mean were excluded from the analyses, causing a loss of $0.2 \%$ of data, across all task conditions and type of sessions. Then, the effect of TMS on the left Ag was assessed conducting a $3 \times 3$ within-subject ANOVA with the repeated-measures factors condition (NC, 
LC and HC) and session-type (no TMS, TMS to left Ag, and TMS to vertex). Bonferroni correction for multiple comparisons was applied on post hoc pairwise contrasts.

\section{Results}

\subsection{Memory task.}

RTs showed a main effect of condition $\left[\mathrm{F}(2,34)=9.443, \mathrm{p}=0.001, \eta \mathrm{p}^{2}=0.357\right]$. Post-hoc comparisons indicated that speed for LC Cue Target condition was slower as compared HC Cue Target $(\mathrm{p}=0.003)$ and Cue Context conditions $(\mathrm{p}=0.009)$. There was no significant difference in speed between the HC Cue Target and Cue Context conditions ( $p$ > 0.999). The main effect of session type was not significant $\left[\mathrm{F}(2,34)=0.188, \mathrm{p}=0.830, \eta \mathrm{p}^{2}=0.011\right]$, suggesting that overall performance was not particularly affected by TMS. However, the results revealed a significant condition $\times$ sessiontype interaction $\left[\mathrm{F}(4,68)=3.446, \mathrm{p}=0.013, \eta \mathrm{p}^{2}=0.169\right]$ that we explored via planned pairwise comparisons. We hypothesised that, in absence of TMS on Ag, the need to update semantic context in LC Cue Target conditions would have induced slower RTs as compared to HC Cue Target conditions. In keeping with this prediction, we found that LC Cue Target conditions were significantly slower than HC Cue Target conditions when TMS was applied to the vertex (control site) $[\mathrm{t}(17)=3.779, \mathrm{p}=0.003$ (adjusted p-value), $\left.d^{\prime}=0.89\right]$ or when it was not applied at all [t $(17)=2.613, \mathrm{p}=0.05$ (adjusted $\mathrm{p}$ value), $\left.d^{\prime}=0.61\right]$ (see Figure 3). We also hypothesised that Ag TMS would eliminate this effect by affecting the HC Cue Target conditions specifically. In accord with our predictions, the LC>HC effect was not observed when TMS was applied to the left $\mathrm{Ag}[\mathrm{t}(17)=0.227, \mathrm{p}>0.999$ (adjusted $\mathrm{p}$-value), $\left.d^{\prime}=0.053\right]$. This was likely due to fact that HC Cue Target conditions became slower when TMS was applied on the left Ag than when it was delivered to the vertex $[\mathrm{t}(17)=2.463, \mathrm{p}=0.05$ (adjusted $\mathrm{p}$ value), $\left.d^{\prime}=0.58\right]$ or when it was not delivered at all $\left[\mathrm{t}(17)=2.576, \mathrm{p}=0.04\right.$ (adjusted $\mathrm{p}$-value), $d^{\prime}=$ 0.607]. Finally, TMS to the left Ag did not impair RT performance for LC Cue Target conditions [all ps $>0.999$ ] or context cue conditions [all ps $>0.42]$. 
The accuracy data did not reveal any significant effects (main effect of condition $[\mathrm{F}(2,34)=$ 2.087, $\left.\mathrm{p}=0.140, \eta \mathrm{p}^{2}=0.109\right] ;$ main effect of session-type $\left[\mathrm{F}(2,34)=0.515, \mathrm{p}=0.602, \eta \mathrm{p}^{2}=0.029\right]$; condition $\times$ session-type interaction $\left.\left[\mathrm{F}(4,68)=0.030, \mathrm{p}=0.998, \eta \mathrm{p}^{2}=0.002\right]\right)$.

In summary, we found that TMS over the left Ag selectively impaired RT performance for the retrieval of contextual information for highly coherent narratives (HC Cue Target conditions).

Please, insert here Figure 3

\subsection{Reading Task.}

Reading times for the target passage showed a main effect of condition $[\mathrm{F}(2,34)=10.806, \mathrm{p}$ $\left.<0.001, \eta \mathrm{p}^{2}=0.389\right]$. Post-hoc comparisons revealed that performance in the NC condition was overall slower than in the $\mathrm{HC}$ and $\mathrm{LC}$ conditions $(\mathrm{p}<0.001$ and $\mathrm{p}=0.037$, respectively). There was no significant RT difference for reading the target paragraph after the HC or LC contexts $(\mathrm{p}=0.257)$. Finally, there was no significant effect of type of session $\left[\mathrm{F}(2,34)=0.551, \mathrm{p}=0.581, \eta \mathrm{p}^{2}=0.031\right]$, or significant a condition $\times$ session-type interaction $\left[F(4,68)=0.231, p=0.920, \eta p^{2}=0.013\right]($ see Figure 4).

Please, insert here Figure 4 


\section{Discussion}

In the present study, we sought evidence for the hypothesis that the left Ag is critical for information buffering and context formation (Humphreys and Lambon Ralph, 2015, 2017; Branzi et al., 2019). This was tested by asking participants to read short narratives consisting of two sequential paragraphs (context and target) and by delivering TMS pulses over the left Ag between the context and target paragraphs, to disrupt online buffering and memory encoding of the narrative content (context and target integrated representation).

In the memory task, we measured RTs and proportion of correct responses to test the hypothesis that TMS-induced disruption of Ag activity during reading (encoding) would have had an effect on formation and therefore recall of integrated memory representation (context-target). We hypothesised that this effect would have been observed for $\mathrm{HC}$ conditions specifically, that is, when incoming information (target) matches the current knowledge-based schema (Speer and Zacks, 2005; Swallow et al., 2009; Humphreys and Lambon Ralph, 2015; van der Linden et al., 2017). In line with this hypothesis, we found that TMS delivered over left Ag made participants slower in retrieving contextrelated information during the memory task, only for coherent narratives (HC Cue Target conditions).

The results presented in this study fit well with the proposal that the core function of the left Ag would be that of an online automatic buffer (Humphreys and Lambon Ralph, 2015, 2017; Humphreys et al., 2019). This portion of the Ag, which is part of the default mode network (DMN), is positively engaged in semantic tasks, but only when these require context-dependent integration (Lerner et al., 2011; Simony et al., 2016; Baldassano et al., 2018; Branzi et al., 2019). Indeed, the very same parietal region is generally deactivated during semantic tasks (Visser and Ralph, 2011; Humphreys et al., 2015), casting some doubts about its key role in semantic integration. On the other hand, however, the left Ag is positively activated by tasks requiring episodic processing (Ramanan et al., 2018; Rugg and King, 2018). 
One common aspect between the above mentioned semantic and episodic tasks is that they both likely require manipulation, retrieval and integration of episodic details (contextual who, what, when, where, why knowledge). In light of this observation, it is possible to relate to the involvement of the left Ag in our and previous studies to at least two mnemonic mechanisms. One would see the left $\mathrm{Ag}$ directly involved in the formation and representations of episodes (e.g., Shimamura, 2011; Bonnici et al., 2016, see the Contextual Integration Model proposed by Ramanan et al., 2018). This hypothesis fits with a series of findings that have shown that the left Ag's activity is prominently observed at the end of an event, i.e., when all the information has been already presented (Humphries et al., 2007; Baldassano et al., 2017). Alternatively, the left Ag may be buffering the combination of past information (episodically retrieved) with newly presented information to update the growing contextual meaning. Not only this hypothesis accords with previous neuroimaging evidence (e.g., (Wagner et al., 2005; Vilberg and Rugg, 2008; Humphreys and Lambon Ralph, 2015, 2017; van der Linden et al., 2017; Branzi et al., 2019; Humphreys et al., 2019), but also with a various neuropsychological findings (for a review see Ramanan et al., 2018). In fact, patients with left Ag lesions are not amnesic like patients with hippocampal lesions on standard source and associative memory paradigms. Rather, damage to the left Ag seems to mainly diminish the confidence in recollection and the efficient online control of information (Simons et al., 2008; Simons et al., 2010). Accordingly, in the present study we show that TMS on the left Ag affects RTs, but not accuracy measures, therefore suggesting that disrupting the normal functioning of the left Ag does not cause the key information to be lost. Rather, TMS over the left Ag may have determined the formation of a less vivid and rich representation of a given episode, therefore making it less easy retrieve (see also Yazar et al., 2014).

Putting these results together, we propose that this DMN Ag region is more likely to be involved in the online buffer and use of episodically-recalled information rather than the coding and integration of this information per se (i.e., episodic and semantic representations might be coded in 
other regions, e.g., hippocampal regions and anterior temporal lobe, respectively). Still, the left $\mathrm{Ag}$ would be critical for the online buffering of internal and external information sources, which is a necessary precursor to the generation and the updating of context meaning.

It is worth to mention that, despite our expectation that TMS over left Ag will also impair behavioural performance during the reading task (and especially for $\mathrm{HC}$ conditions), our results did not support this prediction. In fact, we did not observe the expected disruption in reading times induced by Ag TMS. Although reading times have been previously taken as an index of the efficiency of online buffering processes during reading (e.g., Zwaan et al., 1995; Rinck and Weber, 2003; Speer and Zacks, 2005; Radvansky and Copeland, 2010), it is possible that the experimental design used here was not sensitive enough to detect the Ag TMS effect. In fact, in an attempt to allow enough time for our participants to memorise the content of the narratives, we may have allowed too much time for reading. This possibility is in accord with the fact that we did not replicate the LC>HC difference in reading times, previously observed in an fMRI study in which we allowed less time to respond (Branzi et al., 2019).

To conclude, our findings provide the first evidence of a causal role of the left $\mathrm{Ag}$ in information buffering and context formation during language processing and suggest that Ag activity observed in this and previous studies (e.g., Humphries et al., 2007; Baldassano et al., 2017; Branzi et al., 2019) is causally related to such processes. In fact, the TMS effect observed for HC Cue Target conditions only could not be explained as general episodic memory impairment.

Finally, in the memory task we also found slower RTs for LC Cue Target conditions as compared to HC Cue Target conditions. This result is in accord with previous studies that have shown that memory performance is affected by "event boundaries" (Swallow et al., 2009). In previous studies event boundaries were identified as changes in temporal, spatial and personal dimensions in the course of information processing. To our knowledge, this is the first study to demonstrate that also "semantic 
bioRxiv preprint doi: https://doi.org/10.1101/841478; this version posted November 14, 2019. The copyright holder for this preprint (which was not certified by peer review) is the author/funder. All rights reserved. No reuse allowed without permission.

boundaries", i.e., instances in which a change of semantic context occur, impair the formation and recall of integrated representations. 


\section{References}

Baldassano C, Hasson U, Norman KA (2018) Representation of Real-World Event Schemas during Narrative Perception. J Neurosci 38:9689-9699.

Baldassano C, Chen J, Zadbood A, Pillow JW, Hasson U, Norman KA (2017) Discovering Event Structure in Continuous Narrative Perception and Memory. Neuron 95:709-721 e705.

Binder JR, Desai RH, Graves WW, Conant LL (2009) Where is the semantic system? A critical review and meta-analysis of 120 functional neuroimaging studies. Cereb Cortex 19:27672796.

Bonnici HM, Richter FR, Yazar Y, Simons JS (2016) Multimodal Feature Integration in the Angular Gyrus during Episodic and Semantic Retrieval. J Neurosci 36:5462-5471.

Branzi FM, Humphreys GF, Hoffman P, Lambon Ralph MA (2019) Revealing the neural networks that extract conceptual gestalts from continuously evolving or changing semantic contexts. bioRxiv:666370.

Humphreys GF, Lambon Ralph MA (2015) Fusion and Fission of Cognitive Functions in the Human Parietal Cortex. Cereb Cortex 25:3547-3560.

Humphreys GF, Lambon Ralph MA (2017) Mapping Domain-Selective and Counterpointed Domain-General Higher Cognitive Functions in the Lateral Parietal Cortex: Evidence from fMRI Comparisons of Difficulty-Varying Semantic Versus Visuo-Spatial Tasks, and Functional Connectivity Analyses. Cereb Cortex 27:4199-4212.

Humphreys GF, Jackson RL, Lambon Ralph MA (2019) Overarching principles and dimensions of the functional organisation in the inferior parietal cortex. bioRxiv:654178. 
Humphreys GF, Hoffman P, Visser M, Binney RJ, Lambon Ralph MA (2015) Establishing taskand modality-dependent dissociations between the semantic and default mode networks. Proc Natl Acad Sci U S A 112:7857-7862.

Humphries C, Binder JR, Medler DA, Liebenthal E (2007) Time course of semantic processes during sentence comprehension: an fMRI study. Neuroimage 36:924-932.

Lerner Y, Honey CJ, Silbert LJ, Hasson U (2011) Topographic mapping of a hierarchy of temporal receptive windows using a narrated story. J Neurosci 31:2906-2915.

Oldfield RC (1971) The assessment and analysis of handedness: the Edinburgh inventory. Neuropsychologia 9:97-113.

Radvansky GA, Copeland DE (2010) Reading Times and the Detection of Event Shift Processing. J Exp Psychol Learn 36:210-216.

Ramanan S, Piguet O, Irish M (2018) Rethinking the Role of the Angular Gyrus in Remembering the Past and Imagining the Future: The Contextual Integration Model. Neuroscientist $24: 342-352$.

Rinck M, Weber U (2003) Who when where: An experimental test of the event-indexing model. Mem Cognition 31:1284-1292.

Rossini PM et al. (2015) Non-invasive electrical and magnetic stimulation of the brain, spinal cord, roots and peripheral nerves: Basic principles and procedures for routine clinical and research application. An updated report from an I.F.C.N. Committee. Clin Neurophysiol 126:1071-1107.

Rugg MD, Vilberg KL (2013) Brain networks underlying episodic memory retrieval. Curr Opin Neurobiol 23:255-260. 
Rugg MD, King DR (2018) Ventral lateral parietal cortex and episodic memory retrieval. Cortex 107:238-250.

Sandrini M, Umilta C, Rusconi E (2011) The use of transcranial magnetic stimulation in cognitive neuroscience: A new synthesis of methodological issues. Neurosci Biobehav R 35:516536.

Seghier ML (2013) The angular gyrus: multiple functions and multiple subdivisions. Neuroscientist 19:43-61.

Shimamura AP (2011) Episodic retrieval and the cortical binding of relational activity. Cogn Affect Behav Neurosci 11:277-291.

Simons JS, Peers PV, Mazuz YS, Berryhill ME, Olson IR (2010) Dissociation Between Memory Accuracy and Memory Confidence Following Bilateral Parietal Lesions. Cerebral Cortex 20:479-485.

Simons JS, Peers PV, Hwang DY, Ally BA, Fletcher PC, Budson AE (2008) Is the parietal lobe necessary for recollection in humans? Neuropsychologia 46:1185-1191.

Simony E, Honey CJ, Chen J, Lositsky O, Yeshurun Y, Wiesel A, Hasson U (2016) Dynamic reconfiguration of the default mode network during narrative comprehension. Nat Commun $7: 12141$

Sliwinska MW, James A, Devlin JT (2015) Inferior parietal lobule contributions to visual word recognition. J Cogn Neurosci 27:593-604.

Speer NK, Zacks JA (2005) Temporal changes as event boundaries: Processing and memory consequences of narrative time shifts. J Mem Lang 53:125-140.

Steinmetz H, Furst G, Meyer BU (1989) Craniocerebral topography within the international 10-20 system. Electroencephalogr Clin Neurophysiol 72:499-506. 
Swallow KM, Zacks JM, Abrams RA (2009) Event Boundaries in Perception Affect Memory Encoding and Updating. J Exp Psychol Gen 138:236-257.

van der Linden M, Berkers R, Morris RGM, Fernandez G (2017) Angular Gyrus Involvement at Encoding and Retrieval Is Associated with Durable But Less Specific Memories. J Neurosci 37:9474-9485.

Vilberg KL, Rugg MD (2008) Memory retrieval and the parietal cortex: a review of evidence from a dual-process perspective. Neuropsychologia 46:1787-1799.

Vilberg KL, Rugg MD (2012) The neural correlates of recollection: transient versus sustained FMRI effects. J Neurosci 32:15679-15687.

Visser M, Ralph MAL (2011) Differential Contributions of Bilateral Ventral Anterior Temporal Lobe and Left Anterior Superior Temporal Gyrus to Semantic Processes. J Cognitive Neurosci 23:3121-3131.

Wagner AD, Shannon BJ, Kahn I, Buckner RL (2005) Parietal lobe contributions to episodic memory retrieval. Trends Cogn Sci 9:445-453.

Yazar Y, Bergstrom ZM, Simons JS (2014) Continuous theta burst stimulation of angular gyrus reduces subjective recollection. Plos One 9:e110414.

Zwaan RA, Graesser AC, Magliano JP (1995) Dimensions of Situation Model Construction in Narrative Comprehension. J Exp Psychol Learn 21:386-397. 


\section{Figures and tables legend}

Figure 1.Time line of trial presentation during the reading task.

Figure 2. The left Ag stimulation site pinpointed on the MNI cortical template.

Figure 3. Memory task. Results for (A) RTs and (B) Accuracy (proportion of correct responses) for Cue Context, LC Cue Target and HC Cue Target conditions. Error bars correspond to Standard Errors (SEs).

Figure 4. Reading task. Results for reading times for NC, LC and HC conditions. Error bars correspond to SEs.

Table 1. Examples of stimuli for HC and LC conditions (Context and Target paragraphs). The shift of semantic context after LC contexts was expected to be perceived after the critical ambiguous word, here depicted in red.

Table 2. Example of stimuli for Target Cue and Context Cue conditions, assessing the encoding of context-target and context information, respectively. The stimuli presented below refer to the narrative number 1 presented in Table 1. 
from $500 \mathrm{~ms}$ to $1200 \mathrm{~ms}$

(average $=820 \mathrm{~ms}$ )

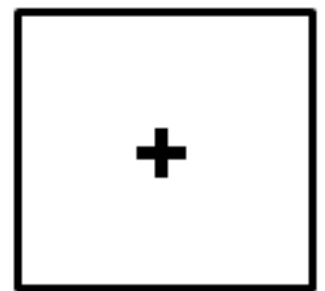

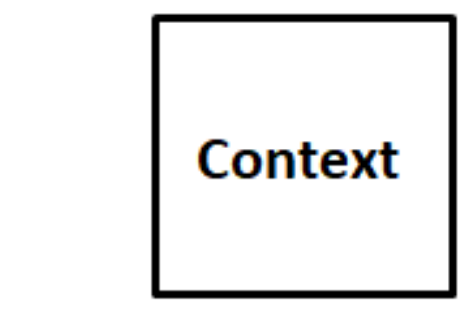

from $3000 \mathrm{~ms}$ to $5460 \mathrm{~ms}$

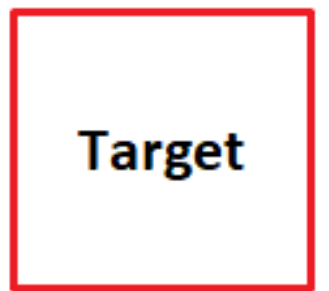

(average $=4230 \mathrm{~ms}$ )
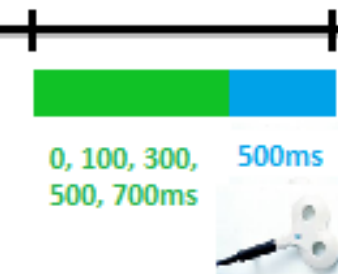

$10 \mathrm{~Hz}, 5$ pulses

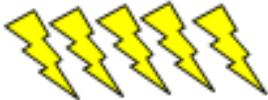


bioRxiv preprint doi: https://doi.org/10.1101/841478; this version posted November 14, 2019. The copyright holder for this preprint (which was not certified by peer review) is the author/funder. All rights reserved. No reuse allowed without permission.

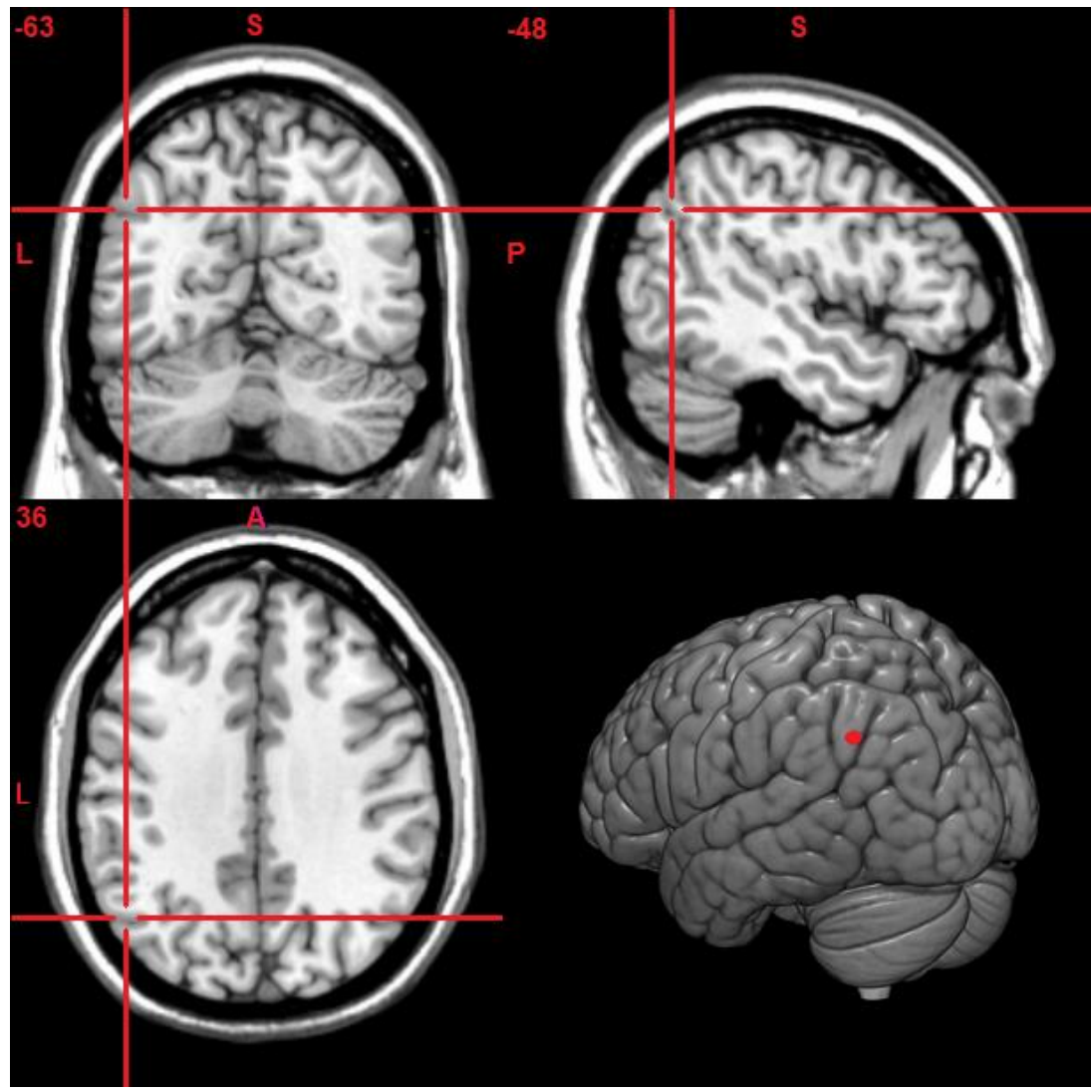



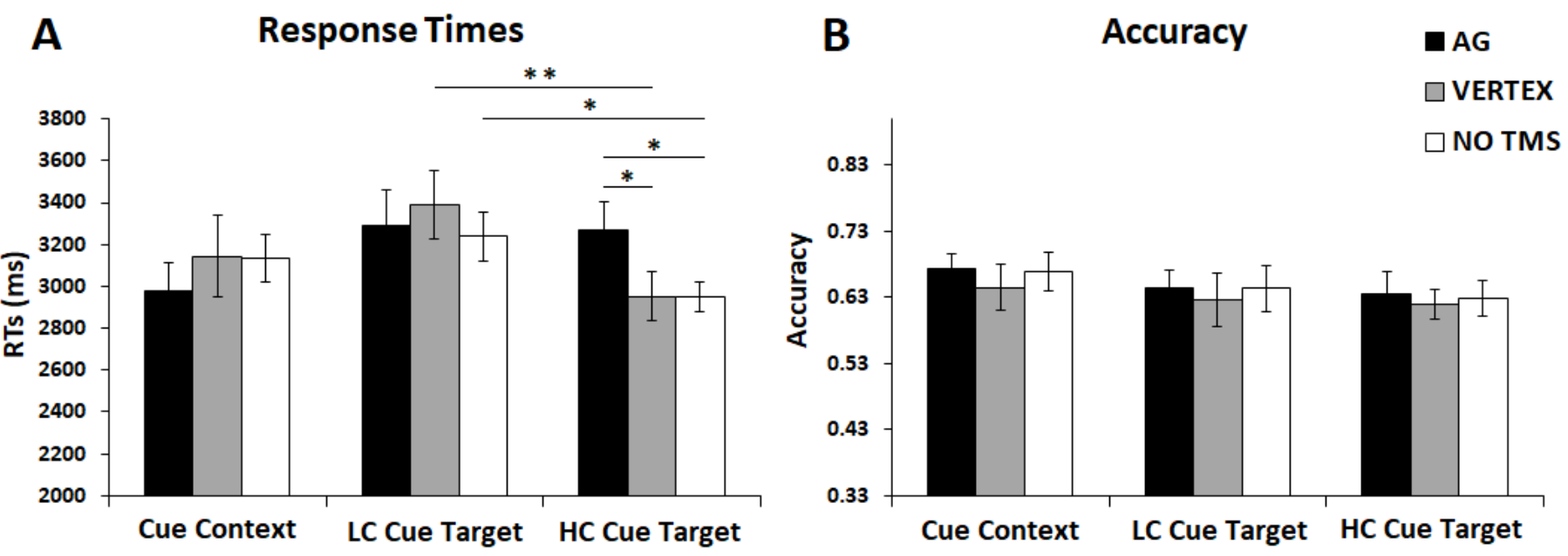


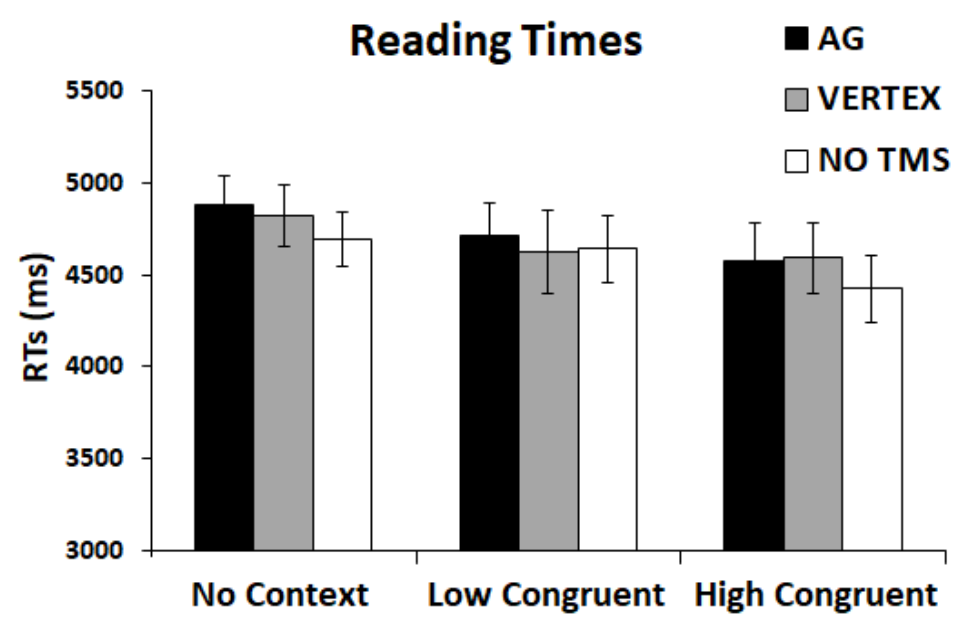


(1) High-congruent context: This famous psychologist was explaining the causes of wrongful convictions throughout history. It seemed that it was a social minority problem. He had to work hard to prove this. Joe was watching the TV very carefully.

(1) Low-congruent context: This famous driver was explaining that his car had a problem which made it slow. It seemed that it was a fuel system problem. The team had to work hard to fix it. Jamie was watching the TV very carefully.

(1) Target: The expert said that race is one aspect of social biases that leads to discriminatory behaviours. This psychologist was saying that these biases can affect people's lives dramatically.

(2) High-congruent context: The garden is a nice place to stay in summer and she spends some time there. She takes care of the flowers and she covers her skin. Unfortunately, she knows very well that it is necessary.

(2) Low-congruent context: The garden is a great place to stay in spring and she spends many hours there. She takes care of the plants and she places traps near the small holes in the ground.

Unfortunately, she knows very well that it is necessary.

(2) Target: Given the large number of moles on her skin she has to be careful with the sun. The doctor told her that she should wear a large hat on her head while she is doing these activities. 


\section{Target Cue conditions (assessing the encoding of context-target information)}

Cue: The expert said that race is one aspect of social biases that leads to discriminatory behaviours. This psychologist was saying that these biases can affect people's lives dramatically.

Question: Where was the psychologist during the interview?

Response Choices: 1 . In a TV studio; 2 . In a radio studio; 3 . In his office

\section{Context Cue conditions (assessing the encoding of context information)}

Cue for LC conditions: This famous driver was explaining that his car had a problem which made it slow. It seemed that it was a fuel system problem.

Cue for HC conditions: This famous psychologist was explaining the causes of wrongful convictions throughout history. It seemed that it was a social minority problem.

Question: Where was the interview?

Response Choices: 1 . In a TV studio; 2 . In a radio studio; 3 . In an office 